\title{
BRAF p.V600E Mutation as Acquired Resistance Mechanism of Lorlatinib in a ROS1 Rearrangement Lung Adenocarcinoma Patient with Primary Ceritinib Resistance
}

\section{Zhulin Liu}

Third Military Medical University Daping Hospital and Research Institute of Surgery

\section{Zhaojie Han}

Southwest Hospital

Naifu Nie

Third Military Medical University Daping Hospital and Research Institute of Surgery

\section{Mingxia Feng}

Third Military Medical University Daping Hospital and Research Institute of Surgery

\section{Li Li}

Third Military Medical University Daping Hospital and Research Institute of Surgery

Yong He ( $\nabla$ heyong@tmmu.edu.cn )

Third Military Medical University Daping Hospital and Research Institute of Surgery

\section{Research Article}

Keywords: ROS1, BRAF p.V600E, lorlatinib resistance, ceritinib resistance

Posted Date: September 14th, 2021

DOI: https://doi.org/10.21203/rs.3.rs-882880/v1

License: (c) (i) This work is licensed under a Creative Commons Attribution 4.0 International License.

Read Full License 


\section{Abstract}

Both crizotinib and ceritinib are recommended as first-line theray for NSCLC with ROS1 rearrangements, with the latter having stronger infiltration ability across blood-brain barrier. Here we report for the first time that a brain metastatic adenocarcinoma patient with CD74-ROS1 rearrangement was sensitive to crizotinib but resistant to ceritinib. Intracranial progression happened after 7 months of crizotinib, and sencond-line lorlatinib resulted in partial response and a PFS of 8 months. Upon lorlatinib resistance, repeated NGS detection of ctDNA from peripheral blood showed BRAF p.V600E, CD74-ROS1 and ROS1 pG2032R. Then, Trametinib, dabrafenib, and cabozantinib was administered to the patient. However, disease progressed very quickly and the patient passed away only one month later, with a overall survival of months. The last ctDNA test, one week before death of the patient, found that BRAF p.V600E disappeared, leaving only CD74-ROS1and ROS1 pG2032R,with a very high frequency. To our knowledge, this is the first case report of a ROS-1 rearrangement lung cancer patient that is sensitive to crizotinib but resistant to ceritinib, and BRAF p.V600E as resistant mechanism for lorlatinib.

\section{Introduction}

The C-ros oncogene 1 receptor kinase (ROS1) rearrangements represent a distinct molecular subtype of non-small cell lung cancer (NSCLC)[1],which occurs mutually exclusive with other known driver gene[2]. The high structural homology especially in the kinase domain between ROS1 and Anaplastic Lymphoma kinase(ALK) was reported[3].Therefore a number of ALK-tyrosine kinase inhibitors (TKIs) retain activity against ROS1 (eg, crizotinib, ceritinib, entrectinib, lorlatinib)[4]. Tumors with ROS1 rearrangements are highly sensitive to ROS1 inhibitors, even better than ALK positive tumors [5].Crizotinib and ceritinib were both the first-line recomended choice in National comprehensive cancer network guidelines (NCCN) version 1.2020 for ROS1 rearrangements NSCLC[6]. Ceritinib demonstrated potent efficiency and safety in patients with ROS1 rearrangements NSCLC who were previously treated with multiple lines of chemotherapy[5]. Besides, a 3rd generation ALK-TKI, lorlatinib, also showed potent efficacy for ROS1 rearrangements NSCLC and G2032R mutation was the most common mechanism for lorlatinib. Here, we report a case of a ROS-1 rearrangement lung cancer patient, who was sensitive to crizotinib but resistant to ceritinib in the first-line setting, and after second-line lorlatinib, BRAF p.V600E together with G2032R emerged as potential resistant mechanism.

\section{Case Description}

We report a case of a 51-year-old Chinese male who was diagnosed with advanced lung adenocarcinoma with spleen, liver, and brain metastasis (stage IVB, T4N2M1c). Next-generation sequencing (NGS) on lung biopsy tissue found CD74-ROS1 rearrangement. For personal reasons, the patient chose crizotinib first.After one month of crizotinib treatment, chest CT scan demonstrated partial response with a decrease in tumor size. Because brain metastasis and ceritinib can cross the blood-brain barrier, the patient demanded a switch to oral ceritinib.However, ceritinib did not show potency and the disease progressed after one month. Fortunately, the tumor shrank again after after the patient resumed 
crizotinib.After 5 months, the intracranial metastases progressed significantly even though the pulmonary lesions remained stable.Lorlatinib, with its strong brain penetration, was given to the patient.Nevertheless, the patient developed psychiatric symptoms, including hallucinations, persecutory delusion, and delirium.At first it's difficult to conclude whether adverse events associated with the central nervous system were due to side effects of lorlatinib or brain metastases. The patient was treated with olanzapine, haloperidol, and mannitol to control psychiatric symptoms, and the dosage of lorlatinib was reduced.MRI of the head indicated that the intracranial metastases had been rapidly relieved and to a profound extent. After the psychiatric symptoms were brought under control, the patient continued to receive lorlatinib due to its strong antitumor ability. The brain metastases were well controlled and no psychiatric symptoms appeared again. However, the disease began to progress after 8 months of lorlatinib treatment.Repeated NGS detection of ctDNA from peripheral blood showed BRAF p.V600E, CD74-ROS1 and ROS1 pG2032R. Trametinib and dabrafenib were used to target BRAF p.V600E mutation, and cabozantinib was administered to inhibit G2032R mutation because the patient declined chemotherapy. However, the disease progressed very quickly including lung lesions and abdominal organ metastases, and the patient died on the date of August 8, 2020.The last ctDNA test on date of August 1 , found that BRAF p.V600E disappeared, leaving only CD74-ROS1 and ROS1 pG2032R, with a very high frequency.

\section{Discussion}

Ceritinib demonstrated potent efficiency and safety in patients with ROS1 rearrangements (including CD74-ROS1 variant) NSCLC, and receives a first-line recommendation in NCCN version 1.2020.As far as we know, this is the first report of the most common form of ROS fusion, CD74-ROS1 variant, that was sensitive to crizotinib but resistant to ceritinib. Therefore, the treatment strategy should not be simply attributed to a sequential application of $1 \mathrm{st}, 2 \mathrm{nd}$, and $3 \mathrm{rd}$ generation TKIs. The cause of primary drug resistance of the CD74-ROS1 variant deserves our attention. In ROS-1 fusion NSCLC patients with ceritinib resistance, crizotinib may be a therapeutic option.In our case, BRAF p.V600E and ROS1 pG2032R were detected in a post-lorlatinib plasma specimen. As previously reported, the most common mechanism of acquired resistance to lorlatinib is G2032R mutation[7]. The newly-detected very low allele frequency of BRAF p.V600E mutation in our case indicates that the mutation evolved during therapy and was subclonal, which may confer a ROS1-independent drug resistance mechanism to ROS-TKIs[8]. After trametinib, dabrafenib, and cabozantinib were given to the patient, the BRAF p.V600E mutation disappeared while the allele frequency of ROS1 pG2032R increased.The ROS1 pG2032R mutation, which was not effectively suppressed by cabozantinib, was the main cause of the poor prognosis of the patient[9].It is suggested that effective inhibition of high abundance drug resistance mechanism may help to improve the prognosis.

\section{Abbreviations}


ALK, Anaplastic Lymphoma kinase; BM, brain metastasis; BSC, best supportive care; EGFR, epidermal growth factor receptor; NGS, next-generation sequencing; LM, liver metastasis; NCCN, National comprehensive cancer network guidelines; NSCLC, non-small cell lung cancer;ORR, objective response rate; $\mathrm{PD}$, progressive disease; PFS, progression-free survival;PR, partial response; ROS1,C-ros oncogene 1 receptor kinase; SD, stable disease; TKI, tyrosine kinase inhibitor

\section{Declarations}

Funding: This work was supported by Daping Hospital, Army Medical Uinversity (2019CXLCA003, 2019CXLCB011), and the Natural Science Foundation of China (under grant numbers 81672284).

Conflicts of interest: none.

Availability of data and material: Not applicable.

Code availability: Not applicable.

Authors' contributions:Zhulin Liu-Conceptualization, Data curation, Investigation,Writing -review \& editing; Zhaojie Han-Data curation, Investigation, Writing -review \& editing; Naifu Nie-Data curation, Investigation,Visualization; Mingxia Feng-Data curation, Investigation,Visualization;Yong HeConceptualization, Funding acquisition, Project administration, Supervision, Writing - review \& editing;Li $\mathrm{Li}$-Conceptualization, Funding acquisition, Project administration, Supervision, Writing - review \& editing.

Ethics approval: The study was approved by ethics board of Daping Hospital, Army Medical University.

Consent to participate: All procedures performed in studies involving human participants were in accordance with the ethical standards of the institutional and national research committees and with the Helsinki Declaration (as revised in 2013). The authors are accountable for all aspects of the work in ensuring that questions related to the accuracy or integrity of any part of the work are appropriately investigated and resolved.

Consent for publication: Written informed consent was obtained from the patient.

\section{References}

1. Bergethon K, Shaw AT, Ou SH, Katayama R, Lovly CM, McDonald NT, et al. ROS1 rearrangements define a unique molecular class of lung cancers. J Clin Oncol. 2012 Mar 10,30(8):863-70. doi: 10.1200/JCO.2011.35.6345.

2. Kohno T, Nakaoku T, Tsuta K, Tsuchihara K, Matsumoto S, Yoh K, et al. Beyond ALK-RET, ROS1 and other oncogene fusions in lung cancer. Transl Lung Cancer Res. 2015 Apr,4(2):156-64. doi: 10.3978/j.issn.2218-6751.2014.11.11. 
3. Chartier M, Chénard T, Barker J, Najmanovich R. Kinome Render: a stand-alone and web-accessible tool to annotate the human protein kinome tree. PeerJ. 2013 Aug 8,1:e126. doi: 10.7717/peerj.126.

4. Shaw AT, Felip E, Bauer TM, Besse B, Navarro A, Postel-Vinay S, et al. Lorlatinib in non-small-cell lung cancer with ALK or ROS1 rearrangement: an international, multicentre, open-label, single-arm first-inman phase 1 trial. Lancet Oncol. 2017 Dec,18(12):1590-1599. doi: 10.1016/S1470-2045(17)30680-0.

5. Lim SM, Kim HR, Lee JS, Lee KH, Lee YG, Min YJ, et al. Open-Label, Multicenter, Phase II Study of Ceritinib in Patients With Non-Small-Cell Lung Cancer Harboring ROS1 Rearrangement. J Clin Oncol. 2017 Aug 10,35(23):2613-2618. doi: 10.1200/JC0.2016.71.3701.

6. Ettinger DS, Wood DE, Aggarwal C, Aisner DL, Akerley W, Bauman JR, et al. NCCN Guidelines Insights: Non-Small Cell Lung Cancer, Version 1.2020. J Natl Compr Canc Netw. 2019 Dec,17(12):1464-1472. doi: 10.6004/jnccn.2019.0059.

7. Jessica Jiyeong Lin,Resistance to lorlatinib in ROS1 fusion-positive non-small cell lung cancer,Poster session(Board \#377),ASCO 2020.

8. Dagogo-Jack I, Rooney M, Nagy RJ, Lin JJ, Chin E, Ferris LA, et al. Molecular Analysis of Plasma From Patients With ROS1-Positive NSCLC. J Thorac Oncol. 2019 May,14(5):816-824. doi: 10.1016/j.jtho.2019.01.009.

9. Katayama R, Kobayashi Y, Friboulet L, Lockerman EL, Koike S, Shaw AT, et al. Cabozantinib overcomes crizotinib resistance in ROS1 fusion-positive cancer. Clin Cancer Res. 2015 Jan 1,21(1):166-74. doi: 10.1158/1078-0432.CCR-14-1385.

\section{Figures}

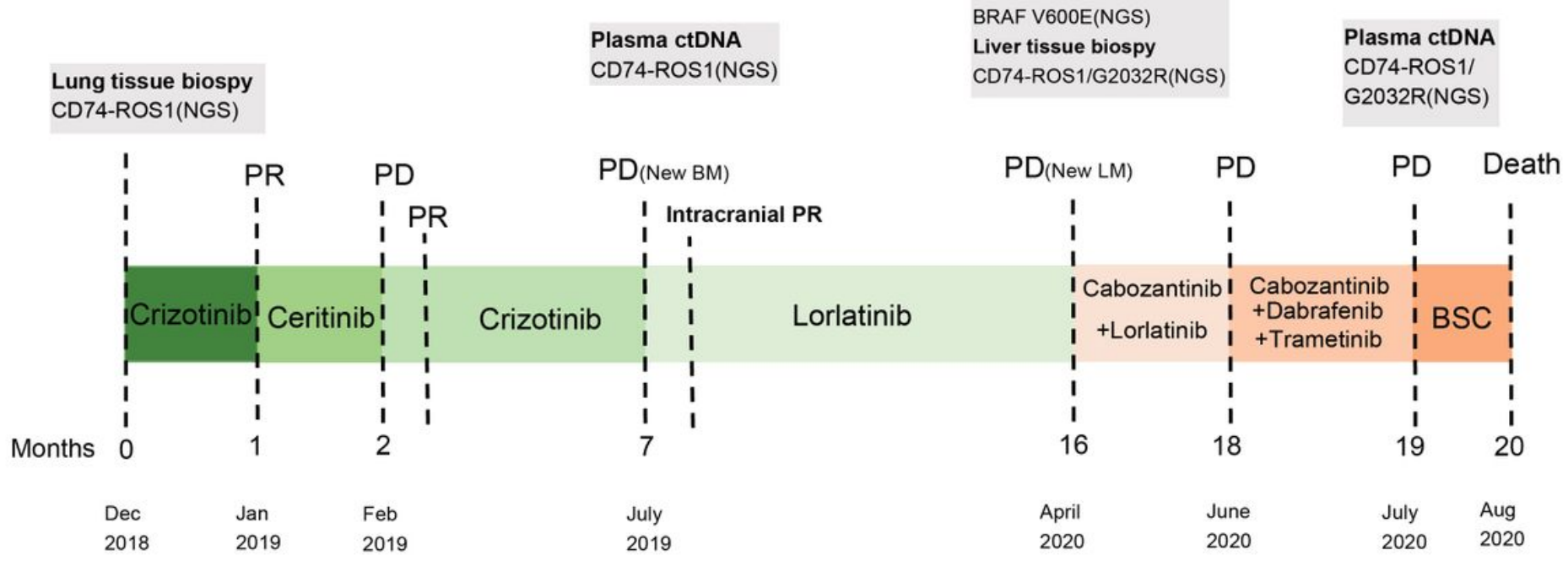

\section{Figure 1}

Schematics showing the timeline of treatment. 


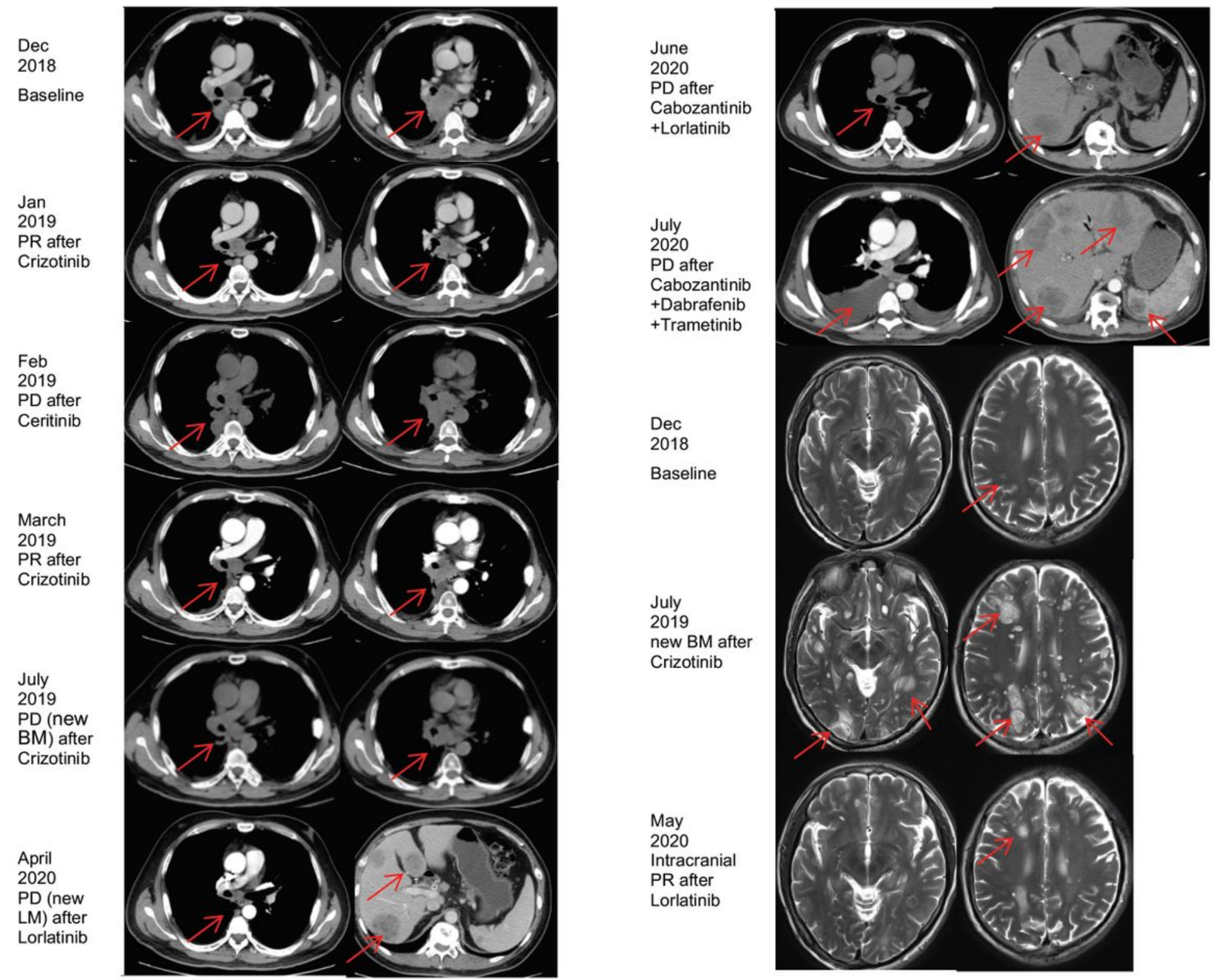

Figure 2

Computed tomography scans revealed clinical response to crizotinib, but PD was occured after ceritinib treatment, and eventually experienced PR again after swiching back to crizotinib. At disease progression following crizotinib treatment, multiple intracranial metastasis were found. 3 weeks after lorlatinib treatment, the patient achieved remarkablely PR of intracranial. Up to 8 months after lorlatinib, the disease was progressed rapidly and lacked of efficiency anti-tumor therapy. 


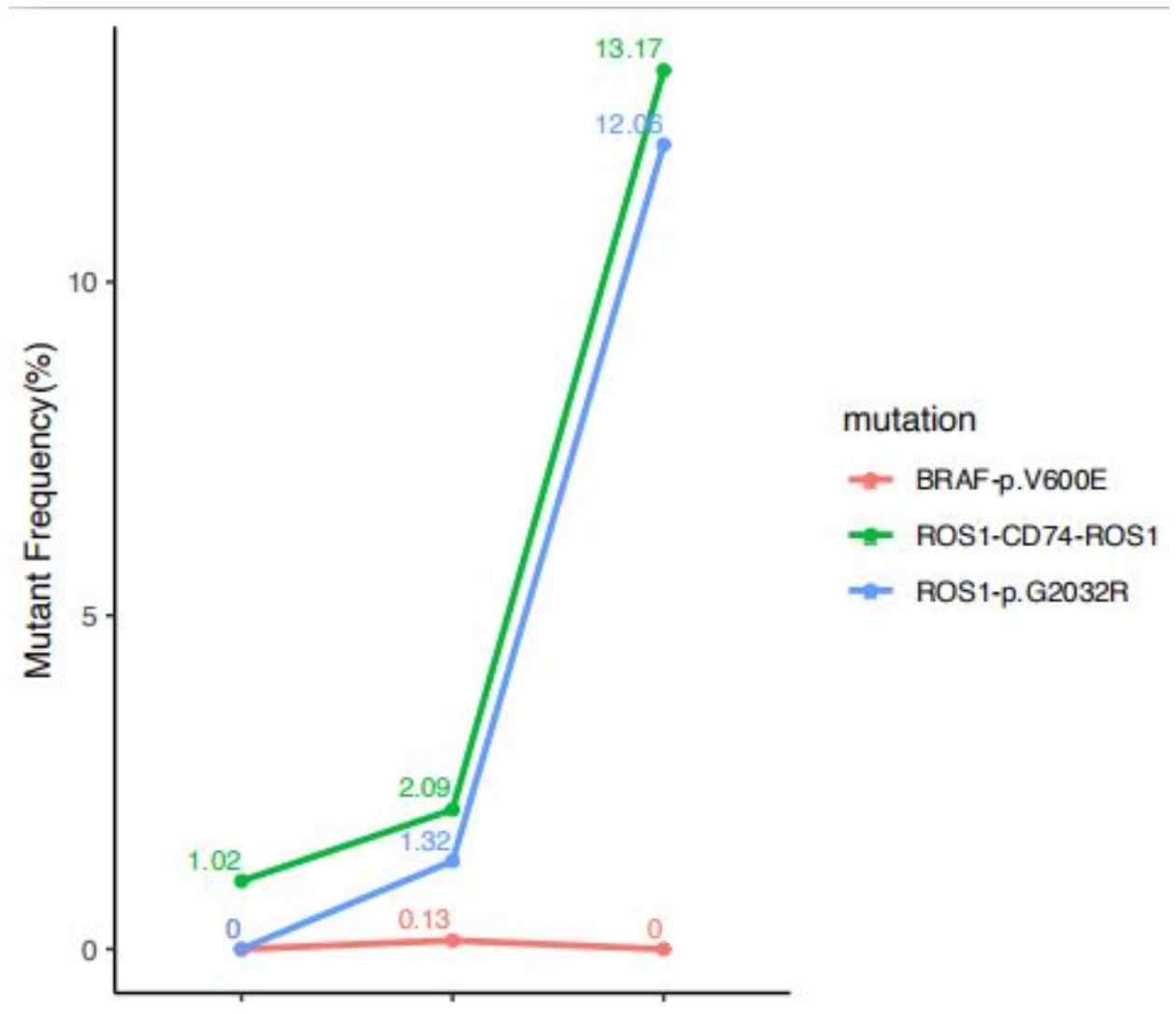

Figure 3

Genetic alterations detected in the patient. 